\title{
On the interpretation of local models in blended multiple model structures*
}

\author{
Robert Shorten, ${ }^{\dagger}$ Roderick Murray-Smith, ${ }^{\ddagger}$ Roger Bjørgan ${ }^{\S}$ and \\ Henrik Golleeף
}

The construction of non-linear dynamics by means of interpolating the behaviour of locally valid models offers an attractive and intuitively pleasing method of modelling non-linear systems. The approach is used in fuzzy logic modelling, operating regime based models, and nonlinear statistical models. The model structure suggests that the composite local models can be used to interpret, in some appropriate manner, the overall non-linear dynamics. In this paper we demonstrate that the interpretation of these local models, in the context of multiple model structures, is not as straightforward as it might initially appear. We argue that the blended multiple model system can be interpreted in two ways - as an interpolation of linearisations, or as a full parameterisation of the system. The choice of interpretation affects experiment design, parameter identification, and model validation. We then show that, in some cases, the local models give insight into full model behaviour only in a very small region of state space. More alarmingly, we demonstrate that for off-equilibrium behaviour, subject to some approximation error, a non-unique parameterisation of the model dynamics exists. Hence, qualitative conclusions drawn from the behaviour of an identified local model, e.g. regarding stable, unstable, nodal or complex behaviour, must be treated with extreme caution. The example of muscle modelling is used to illustrate these points clearly.

\section{Introductory remarks}

The past few years have shown an increase in the use of local model representations of nonlinear dynamic systems (see (Johansen and Murray-Smith 1997) for a review). This basic structure includes a number of approaches: Tagaki-Sugeno fuzzy systems (Takagi and Sugeno 1985), local model networks, gain-scheduled control, statistical mixture mod-

\footnotetext{
* To appear in the International Journal of Control, 1998.

$\dagger$ Dept. of Electronic and Electrical Eng., University College Dublin, Ireland. robert @ faraday • ucd. ie

$\ddagger$ Dept. of Mathematical Modelling, Technical University of Denmark, Lyngby, Denmark. rode imm.dtu.dk

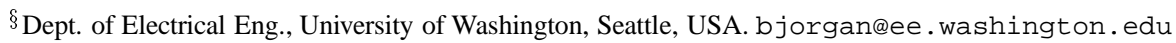

^ Centre for Systems and Control, Glasgow University, Scotland. henrik@eng.gla.ac.uk
} 
els, Smooth Threshold AutoRegressive (STAR) models of Tong (1990) and the State dependent models of Priestley (1988), among them. The model parameters are obtained from prior knowledge, linearisations of a physical model or identified from measured data. Advantages of this approach are purported to be its simplicity and the insight into global dynamics obtained from the local models.

By a blended local model structure we understand a dynamic model of the form

$$
\dot{\mathbf{x}}=\sum_{i}^{N_{m}} \rho_{i}(\mathbf{x}, \mathbf{u}, \mathbf{w}) \mathbf{f}_{\mathbf{i}}(\mathbf{x}, \mathbf{u}),
$$

where state $\mathbf{x} \in \mathbb{R}^{N}$, input $\mathbf{u} \in \mathbb{R}^{P}$, and an external vector $\mathbf{w} \in \mathbb{R}^{O}$, the model $\mathbf{f}_{\mathbf{i}}(:,:)$ is one of $N_{m}$ vector functions of the state and the input, and is valid in a region defined by the scalar validity function $\rho_{i}$, which in turn is a function of the above variables. Typically, the local models $\mathbf{f}_{i}$ are chosen to be of the form $\mathbf{f}_{i}(\mathbf{x}, \mathbf{u})=\mathbf{A}_{\mathbf{i}} \mathbf{x}+\mathbf{B}_{\mathbf{i}} \mathbf{u}+\mathbf{d}_{\mathbf{i}}$, resulting in constituent dynamic systems $\Sigma_{i}$ given by,

$$
\begin{aligned}
\Sigma_{i}: \dot{\mathbf{x}} & =\mathbf{f}_{i}(\mathbf{x}, \mathbf{u}) \\
& =\mathbf{A}_{\mathbf{i}} \mathbf{x}+\mathbf{B}_{\mathbf{i}} \mathbf{u}+\mathbf{d}_{\mathbf{i}},
\end{aligned}
$$

where $\mathbf{x}, \mathbf{d}_{i} \in \mathbb{R}^{N}, \mathbf{A}_{i} \in \mathbb{R}^{N \times N}$, and $\mathbf{B}_{i} \in \mathbb{R}^{N \times P}$. This results in a non-linear description of plant dynamics of the form,

$$
\dot{\mathbf{x}}=\mathbf{A}(\mathbf{x}, \mathbf{u}, \mathbf{w}) \mathbf{x}+\mathbf{B}(\mathbf{x}, \mathbf{u}, \mathbf{w}) \mathbf{u}+\mathbf{d}(\mathbf{x}, \mathbf{u}, \mathbf{w}),
$$

where,

$$
\begin{aligned}
\mathbf{A}(\mathbf{x}, \mathbf{u}, \mathbf{w}) & =\sum_{i}^{N_{m}} \rho_{i}(\mathbf{x}, \mathbf{u}, \mathbf{w}) \mathbf{A}_{\mathbf{i}} \\
\mathbf{B}(\mathbf{x}, \mathbf{u}, \mathbf{w}) & =\sum_{i}^{N_{m}} \rho_{i}(\mathbf{x}, \mathbf{u}, \mathbf{w}) \mathbf{B}_{\mathbf{i}} \\
\mathbf{d}(\mathbf{x}, \mathbf{u}, \mathbf{w}) & =\sum_{i}^{N_{m}} \rho_{i}(\mathbf{x}, \mathbf{u}, \mathbf{w}) \mathbf{d}_{\mathbf{i}}
\end{aligned}
$$

The $\mathbf{A}_{\mathbf{i}}$ matrices associated with each of the local models are assumed to be invertible; that is, associated with each constituent local model there is exactly one unique equilibrium point. Model building thus consists of covering the state space of the non-linear plant with local models. Behaviour along the plant equilibria is typically captured by using models whose equilibria $\left(\mathbf{x}_{i 0}=-\mathbf{A}_{i}^{-1} \mathbf{d}_{i}\right.$, in the unforced case) are located inside the region defined by their basis functions, whereas behaviour off equilibria is captured by using models whose (virtual) equilibria are located outside the region defined by their basis functions (hence the 'virtual' label).

In this paper we demonstrate that the interpretation of local models is not straightforward, and depends both upon the parameters of the validity functions and upon the location of the local model equilibria. 


\section{Interpretation and identification}

Typically, identification of a local model network either involves conventional linearisation of the non-linear system about a number of equilibrium operating points, or performing weighted regression of local models to excitation data (in which case the models are not the classical linearisations commonly used in control theory).

Given these two possibilities, there are two approaches to interpretation of the model at intermediate operating points, where the model description of the plant dynamics is obtained by interpolating the local models in some manner. For example, consider the model of the unforced plant dynamics obtained by identifying linear models about several values of scheduling vector $\left(\mathbf{x}_{i}, \mathbf{w}_{0 i}\right):^{1}$

1. We could interpret the interpolation procedure as yielding intermediate Jacobian matrices given by $\mathbf{A}\left(\mathbf{x}_{0}, \mathbf{w}_{0}\right)$. This results in a model of the linearised dynamics at $\left(\mathbf{x}_{0}, \mathbf{w}_{0}\right)$ given by $\dot{\mathbf{x}}=\mathbf{A}\left(\mathbf{x}_{0}, \mathbf{w}_{0}\right)\left(\mathbf{x}-\mathbf{x}_{0}\right)$.

2. Or, we could assume that the multiple model family used is a parameterisation of the real system yielding a full description of global dynamics $\dot{\mathbf{x}}=\mathbf{A}(\mathbf{x}, \mathbf{w}) \mathbf{x}+\mathbf{d}(\mathbf{x}, \mathbf{w})$. In this case the linearisation about an intermediate equilibrium point $\left(\mathbf{x}_{0}, \mathbf{w}_{0}\right)$ is given by,

$$
\begin{aligned}
\dot{\mathbf{x}}= & \left(\frac{\partial}{\partial x}[\mathbf{A}(\mathbf{x}, \mathbf{w}) \mathbf{x}+\mathbf{d}(\mathbf{x}, \mathbf{w})]\right)_{\left(\mathbf{x}_{0}, \mathbf{w}_{0}\right)}\left(\mathbf{x}-\mathbf{x}_{0}\right)+ \\
& \left(\frac{\partial}{\partial w}[\mathbf{A}(\mathbf{x}, \mathbf{w}) \mathbf{x}+\mathbf{d}(\mathbf{x}, \mathbf{w})]\right)_{\left(\mathbf{x}_{0}, \mathbf{w}_{0}\right)}\left(\mathbf{w}-\mathbf{w}_{0}\right) \\
= & \overline{\mathbf{A}}_{\mathbf{x}}\left(\mathbf{x}_{\mathbf{0}}, \mathbf{w}_{\mathbf{0}}\right)\left(\mathbf{x}-\mathbf{x}_{\mathbf{0}}\right)+\mathbf{A}_{\mathbf{w}}^{-}\left(\mathbf{x}_{\mathbf{0}}, \mathbf{w}_{\mathbf{0}}\right)\left(\mathbf{w}-\mathbf{w}_{\mathbf{0}}\right)
\end{aligned}
$$

where $\overline{\mathbf{A}}_{\mathbf{x}}\left(\mathbf{x}_{0}, \mathbf{w}_{0}\right) \in \mathbb{R}^{\mathbf{N} \times \mathbf{N}}$ and where $\overline{\mathbf{A}_{\mathbf{w}}}\left(\mathbf{x}_{0}, \mathbf{w}_{0}\right) \in \mathbb{R}^{N \times O}$.

If identifying the models from experimental data, the first interpretation implies that the linearisation is based only on perturbation data around the linearisation point $\left(\mathbf{x}_{0}, \mathbf{w}_{\mathbf{0}}\right)$. In the second case (equation (2.1)), we assume global excitation of the input space, and that the local models are not to be identified independently of each other, but rather that the identification of basis functions and local models is performed in an iterative process. We note that $\overline{\mathbf{A}}_{\mathbf{x}}\left(\mathbf{x}_{0}, \mathbf{w}_{0}\right)$ and $\mathbf{A}\left(\mathbf{x}_{0}, \mathbf{w}_{0}\right)$ are in general not identical. To sum up - what the model represents depends on how the data are gathered, and how the parameters of the local models and basis functions are identified.

Example 2.0.1 To illustrate this point more clearly, consider the example depicted in Figure 1. Here, a mathematical model of a helicopter was perturbed ${ }^{2}$ around a number of

\footnotetext{
${ }^{1}$ Note here we have omitted $\mathbf{u}$ from the scheduling variable for simplicity. In the remainder of the paper we shall no longer schedule on an external $\mathbf{w}$ and shall therefore use $\mathbf{A}$ for $\mathbf{A}_{\mathbf{x}}$.

${ }^{2}$ Helicopter linearisation data provided by Stewart Houston, University of Glasgow. The model is too extensive to include in this paper - see (Houston 1994) for further details.
} 
linearisation points $x_{i}$ (scheduling on airspeed, from hover to 10 knots at 1 knot intervals) to provide the parameters for local state-space models, which are then integrated into a multiple model system using locally linear basis functions to form a model of the system dynamics. The use of perturbations around an operating point implies that we are using the first interpretation.

In Figure 1 we show the eigenvalues of the $\mathbf{A}\left(\mathbf{x}_{0}\right)$ and $\overline{\mathbf{A}}\left(\mathbf{x}_{0}\right)$ matrices as defined above. Figure 1(c) shows the linearly interpolating basis functions, and their derivatives. In the second approach the non-zero derivatives of the basis functions, and of the offset terms have a significant effect on the linearisations. In Figure 1(a) we see the poles of the identified A matrices, and interpolation between them provides 'sensible' results. These A matrices already implicitly contain the extra terms described in the second interpretation. If we interpret them wrongly by adding in the effect of basis function derivatives and change of offset, we get meaningless interpolation, as shown in Figure 1(b), where the full model eigenvalues do not even pass throught the local model eigenvalues at the linearisation points (because of the nonzero derivative of the basis function, $\frac{\partial \rho_{\mathbf{i}}(\mathbf{x})}{\partial \mathbf{x}}$ ). Adding further local models would not improve matters.

This example illustrates the care which should be taken when interpreting the parameters of a multiple model system. ${ }^{3}$ This obviously has to be taken into account when dealing with grey-box models which combine identified and a priori components, as well as for experiment design, identification algorithms, and any subsequent control design.

\section{Interpretation of model dynamics}

The structure of the non-linear system (1.4) and the manner in which it is identified encourage a certain interpretation of the model dynamics; namely to interpret the model dynamics in terms of the individual composite dynamic systems $\Sigma_{i}$. In fact, the structure is such that it is quite tempting to interpret the quantitative behaviour of the model dynamics in terms of the poles and zeros of $\Sigma_{i}$. Such an interpretation is not generally valid for a number of reasons. Apart from the fact that the eigenvalues of the parameterised matrix $\mathbf{A}(\mathbf{x}, \mathbf{u}, \mathbf{w})$ depend not only on the local models $\mathbf{A}_{i}, \mathbf{B}_{i}, \mathbf{d}_{i}$, but also upon the interpolation procedure, several other problems exist which invalidate this interpretation. In particular we note the following important observations which provide the basis for the remaining discussion in this paper.

(i) Local models along the manifold of equilibria are only individually interpretable in a region where $\rho_{i} \approx 1 .{ }^{4}$

\footnotetext{
${ }^{3}$ Note that one could argue for a variety of implementations of the basis function and local models for this case - the example is purely to illustrate the relevance of the points discussed above.

${ }^{4}$ For the second interpretation (equation (2.1)), we see that $\overline{\mathbf{A}}_{\mathbf{x} i}(\mathbf{x}) \approx \mathbf{A}_{i}$ only if $\rho_{i} \approx 1$ and $\frac{\partial \rho_{\mathrm{i}}(\mathbf{x})}{\partial \mathbf{x}} \approx 0$. Hence, the effect of the derivative may contribute significantly to the linearisation term unless the neighbouring $\mathbf{A}_{i}$ 's are identical.
} 


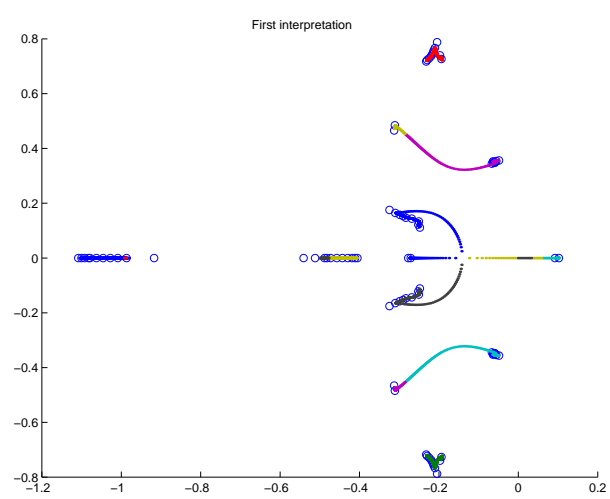

(a) Eigenvalues of $\mathbf{A}\left(\mathbf{x}_{0}\right)$ are marked by dots. Eigenvalues of linearisations $\mathbf{A}_{i}$ are circles.

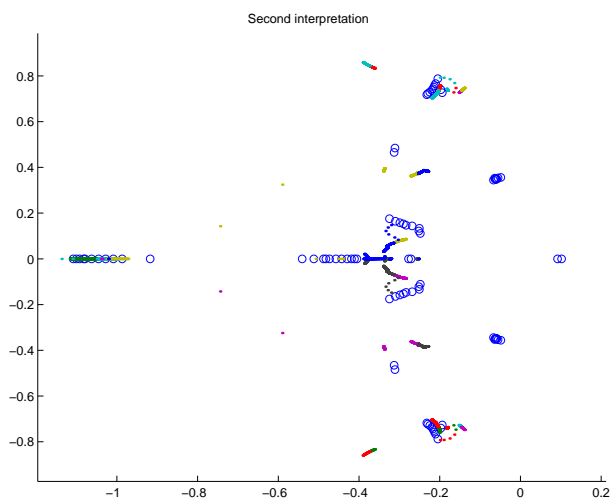

(b) Eigenvalues of $\overline{\mathbf{A}}\left(\mathbf{x}_{0}\right)$ are marked by dots. Eigenvalues of linearisations $\mathbf{A}_{i}$ are marked by circles. Note the divergence from the interpolated eigenvalues in (a), and that the interpolated eigenvalues no longer pass through the identified eigenvalues, even at the linearisation points.
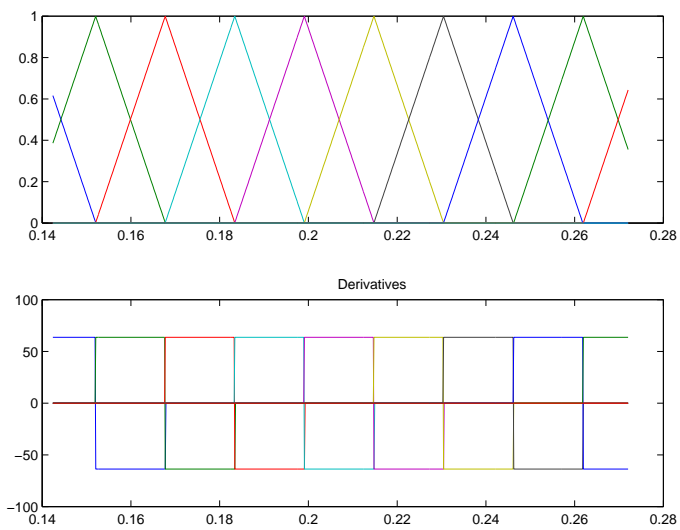

(c) Linear interpolation basis functions of local model net, and their derivatives. Basis functions have compact support. Basis functions are plotted for the region used for the interpolation plots in a) and b). The $x$-axis is normalised, with hover from the left to 10 knots on the right.

Figure 1.. Illustration of the effect of model interpretation in a practical example. The local models were linearisations based on perturbations of a helicopter model from a trimmed state, at 1 knot intervals from hover to 10 knots. Eigenvalues of individual local models are shown, along with interpolated values from the multiple model structure. 
(ii) Off equilibria, for models with virtual equilibrium points, only part of the dynamics associated with model $\Sigma_{i}$ is used in the construction of the global dynamics. Hence, the region of validity of the model is more restricted than that defined by its blending function $\rho_{i}$. The model is only valid in a subspace of this region.

Both of these observations, and their consequences for interpreting stuctures such as the local model network (Johansen and Murray-Smith 1997) will now be discussed.

\subsection{Interpretation problems of on-equilibrium local models}

It is well known that instability and even chaotic behaviour can be introduced by switching or interpolating between stable linear systems (Fillipov 1960, Skoog and Clifford 1972, Shorten 1996). Hence, the dynamics of the individual sub-systems may, in some cases, give no useful insight into the global system dynamics. In the case of equation (1.4) such effects depend upon the validity functions and linearisation points (hence the $\mathbf{d}_{i}$ ) - the local linearisations are often only indicative of global dynamics in a small region where $\rho_{i} \approx 1$, around their equilibrium points. In this region, the global model behaves approximately as the local model, and returns to the equilibrium point. As we leave this region we may see periodic oscillatory behaviour, or chattering between neighbouring local models. Further still, and the system state may leave the local model's regime completely. This will often in fact be desirable behaviour, but it renders interpretation of the individual local models meaningless in many cases.

\subsection{Interpretation problems of off-equilibrium local models}

A further factor which affects our interpretation of multiple model dynamics concerns off-equilibrium behaviour. Consider the phase-plane trajectory depicted in Figure 2. It can be observed that the trajectory depicted may be approximated by combining models as shown in Figure 2 (a) or as in Figure 2 (b). This suggests that the state space can be covered by many different locally accurate models which, when combined, will approximate this trajectory in a satisfactory manner. To see this more clearly consider the abstract case of approximating the flow associated with the dynamic system $\dot{\mathbf{x}}=\mathbf{f}(\mathbf{x})$, in the vicinity of some vector $\mathbf{x}_{0}$ by the local model

$$
\dot{\mathbf{x}}=\rho(\mathbf{x})[\mathbf{A} \mathbf{x}+\mathbf{d}]
$$

where $\mathbf{f}(:) \in \mathbb{R}^{\mathbf{N}}$, where $\mathbf{x}, \mathbf{A}, \mathbf{d}$ are as defined in Section 1., and where $\rho(\mathbf{x})=1$ when $\mathbf{x}=\mathbf{x}_{0}$, and is zero otherwise. Clearly for any arbitrary choice of invertible $\mathbf{A}$, regardless of its nature (stable, unstable, complex, etc.), a vector $\mathbf{d}$ can be found such that

$$
\mathbf{f}\left(x_{0}\right)=\rho\left(\mathbf{x}_{0}\right)\left[\mathbf{A} \mathbf{x}_{0}+\mathbf{d}\right]
$$

where $\mathbf{d}=\mathbf{f}\left(\mathbf{x}_{0}\right)-\mathbf{A} \mathbf{x}_{0}$. Hence, at $\mathbf{x}=\mathbf{x}_{0}$ a non-unique parameterisation of the dynamics exist, and indeed the linearisation is meaningless. Furthermore, in the neighbourhood 


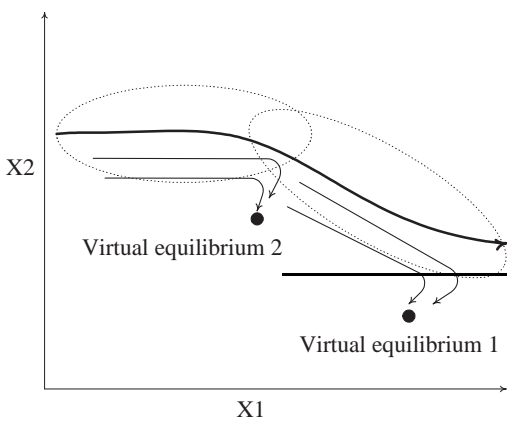

(a) Model construction.

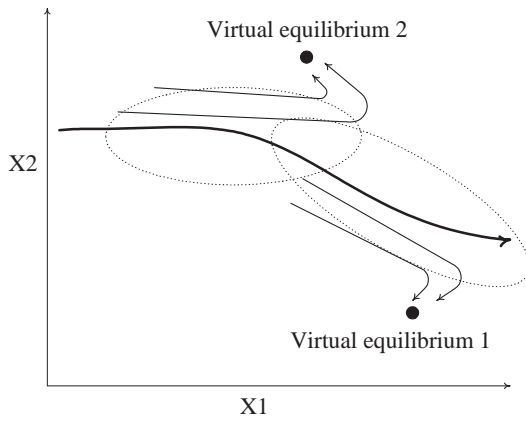

(b) Alternative model construction.

Figure 2.. Construction of a trajectory using interpolated local models. The ellipses denote the location of the $\rho_{i}$. Note how alternative models' trajectories approximate the measured trajectory within their basis functions, but diverge significantly outside the basis function. This observation has severe consequences for control law design.

of $\mathbf{x}_{\mathbf{0}}$, subject to some approximation error, by simply varying the location of the virtual equilibria (or the form of the $\rho_{i}$ ), it is possible to obtain many (dynamically) different parameterisations of the non-linear dynamics. ${ }^{5}$ This is illustrated in the following example.

Example 3.2.1 Consider the behaviour of the following autonomous systems,

$$
\begin{aligned}
\Sigma_{1}: \dot{\mathbf{x}} & =\mathbf{A}_{\mathbf{1}} \mathbf{x}, \\
\Sigma_{2}: \dot{\mathbf{x}} & =\mathbf{A}_{\mathbf{2}} \mathbf{x}+\mathbf{d},
\end{aligned}
$$

where $\mathbf{A}_{\mathbf{1}}=\left[\begin{array}{cc}4 & -4.5 \\ 4.05 & -4.55\end{array}\right], \mathbf{A}_{\mathbf{2}}=\left[\begin{array}{cc}0.51 & -4.29 \\ 3.84 & -4.51\end{array}\right]$, and $\mathbf{d}=\left[\begin{array}{c}-8.58 \\ -0.27\end{array}\right]$.

The flow associated with both of these systems is depicted in Figure 3. These systems are qualitatively very different; $\Sigma_{1}$ is a stable node with an equilibrium point centered at the origin, whereas $\Sigma_{2}$ is a stable spiral with its equilibrium point close to, but not centered, at the origin. However, in a small region defined by,

$$
R: 2 \leq x_{1} \leq 4,8 \leq x_{2} \leq 10,
$$

as depicted, the flow of both systems is similar. The velocity vectors point in the same direction and the maximum error,defined by,

$$
\epsilon_{\max }=\max _{x \in R} \frac{\left\|\left(\mathbf{A}_{\mathbf{2}}-\mathbf{A}_{\mathbf{1}}\right) \mathbf{x}+\mathbf{d}\right\|}{\|\mathbf{x}\|},
$$

\footnotetext{
${ }^{5}$ We note also that conditions exist such that two systems, which have the same equilibrium point, can be identical along an entire manifold; namely, when $\mathbf{A}_{1}$ and $\mathbf{A}_{\mathbf{2}}$ share eigenvector and eigenvalue pairs. The manifold is defined by the eigenvectors common to both systems.
} 


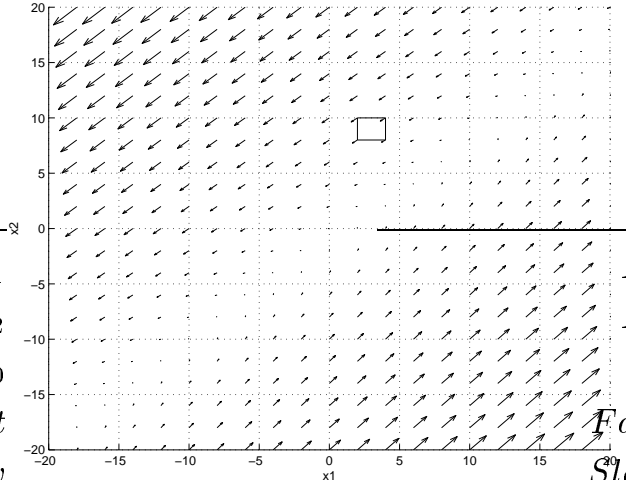

(a) Dynamics associated with $\Sigma_{1}$

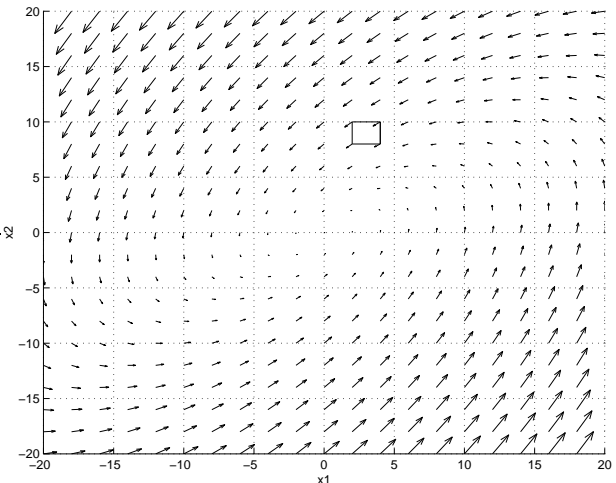

(b) Dynamics associated with $\Sigma_{2}$

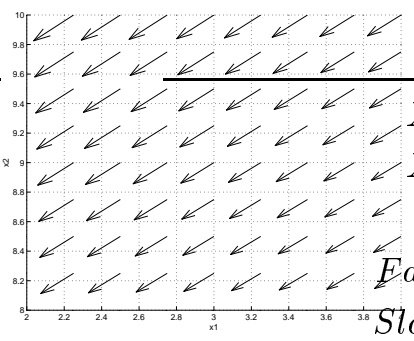

(c) The region $R 1$.

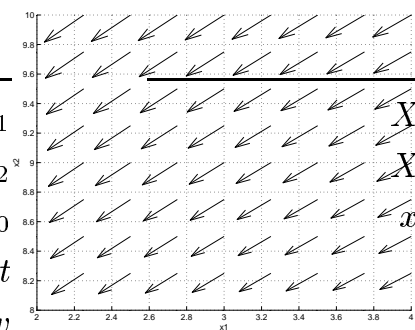

(d) The region $R 2$

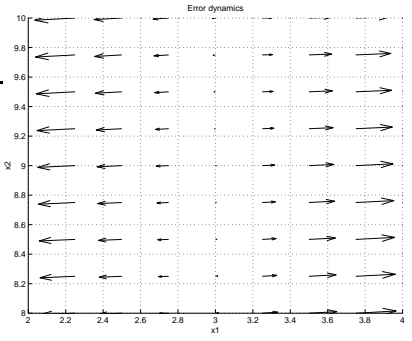

(e) Error dynamics

Figure 3.. Non-uniqueness of representation. The two systems $\Sigma_{1}$ and $\Sigma_{2}$ are qualitatively different, but in the outlined regions (Shown in detail in (c) and (d)) we see that there is little difference, as shown by the error dynamics in (e). 


$$
<0.42
$$

is bounded and small. The error dynamics

$$
\dot{\mathbf{x}}=\left(\mathbf{A}_{\mathbf{2}}-\mathbf{A}_{\mathbf{1}}\right) \mathbf{x}+\mathbf{d}, \mathbf{x} \in R,
$$

are depicted in Figure 3(e). Hence, we conclude that in $R$, subject to some appropriately defined approximation error, the dynamics described by $\Sigma_{A_{1}}$ and $\Sigma_{A_{2}}$ are in some sense equivalent. In this region both $\Sigma_{A_{1}}$ and $\Sigma_{A_{2}}$ are valid representations of an appropriate non-linear system, but outside the region they differ considerably.

This rather obvious observation is of crucial importance for two reasons. It strongly suggests that the qualitative nature of the identified local models may say very little about the non-linear dynamics even locally. This is by virtue of the fact that the local model is, by definition, only valid in a local region of state space, and crucially in the off-equilibrium case, that the local model's contribution to the global model only comes from a restricted sub-region which does not include the model's equilibrium point. Given enough data, from a well-designed experiment, we could avoid such problems, as we would know we had covered the volume of interest in the input space. In practice though, where we have too poor understanding of the target system to design an ideal experiment, and where exhaustive data acquisition is too expensive, this will often be a real problem.

Secondly, given the variability in possible solutions to the identification problem, we may wish to regularise the identification process, that is bias the choice from the space of possible fits to the data towards models with desired properties (for example stable local linear models). A simple alternative, especially if we only want on-equilibrium models, so it is known a priori that local models should have 'real' equilibria, is to identify offset models $\mathbf{d}_{i}()$ separately from dynamics models. This is a practical approach, as a wide class of systems can be easily driven through a range of equilibria to acquire the necessary data, and at a finer quantisation level than in experiments linked to the dynamic behaviour. Straightforward interpolation with smoothing provides the model, and validation is also straightforward. If we then use this model of the offsets as the basis for the linearisations we have severly reduced the degrees of freedom for the linear system.

\section{Modelling muscle behaviour}

An example of identifying a local model with a virtual equilibrium point, and being able to correct problems (in this case 'by hand'), was found when modelling isometric contraction of electrically stimulated rabbit muscle (Gollee et al. 1997). The motoneurons of the muscle are stimulated with randomly spaced impulses, and the force produced by the muscle when held at constant length is recorded. Typical data are shown in Figure 4(a). The system has a single input and a single output.

A model of the form of equation (1.4) with six local linear second order models is identified using 30 data sets, where each set contains 590 samples. The parameters of 

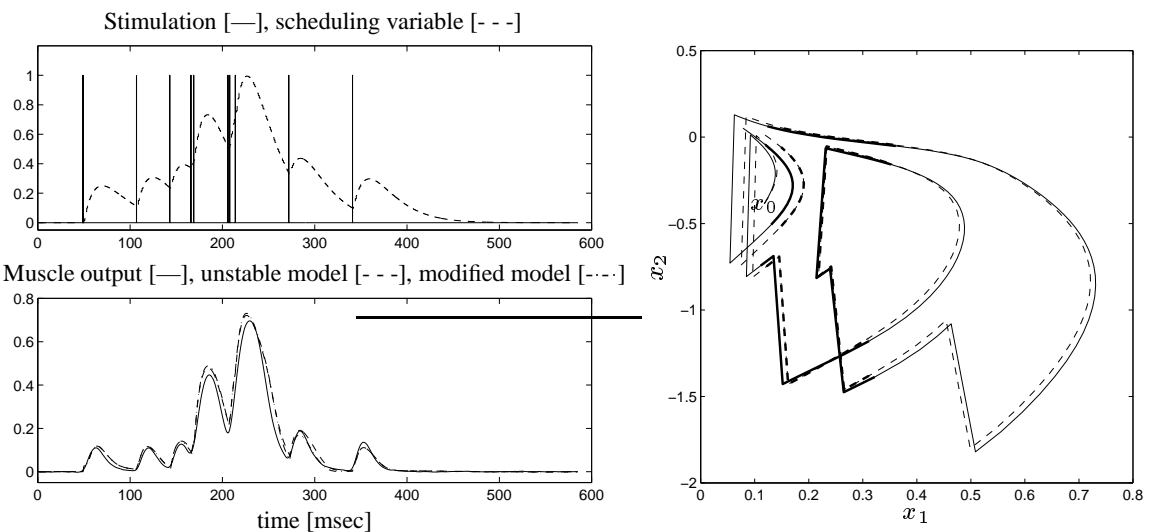

(a) Model behaviour over time. The responses of

(b) Phase plane trajectories of the unstable and the modified model shown in Figure 4(a) from 150 to $300 \mathrm{~ms}$. Bold line indicates that the fourth local model is active $\left(\rho_{4}>0.3\right)$. The sudden vertical jumps in $\mathbf{x}$ are due to the input impulses shown in Figure 4(a).

Figure 4.. Experimental data and model responses.

the local models were optimised using a Levenberg-Marquardt algorithm with an infinite prediction horizon (Press et al. 1992). As shown in Figure 4(a), the performance of the global model is very good for stimulation sequences similar to those used to identify its parameters, which consist of pulses with randomly varying inter-pulse interval. If we examine the identified $\mathbf{A}$ matrices, we find that all have real and negative eigenvalues, except for the fourth local model which has eigenvalues $7.14,-111.89$, i.e. this local model has a non Hurwitz A matrix, which is undesirable - if the model enters a region where we had no identification data, a Hurwitz matrix will tend to push us toward equilibria and thus hopefully into a more accurately modelled region. ${ }^{6}$ All local models, other than the one scheduled at $w=0$ have virtual equilibria. Given the stimulation the model was identified with, where the activation varies quickly, and each local model remains activated only for a short period of time, this slow positive eigenvalue did not have time to have an effect. However, the influence of the unstable local model becomes obvious when a constant frequency burst is applied which drives the model into an operating region where the unstable local model is constantly active. Such a response is shown in Figure 5(a).

To show how a range of models can fit the data locally, but have quite different properties, we altered the positive eigenvalue to give us a stabilised model with a slow but stable

${ }^{6}$ It could also lead to limit cycle behaviour, but should not lead to unboundedness. 

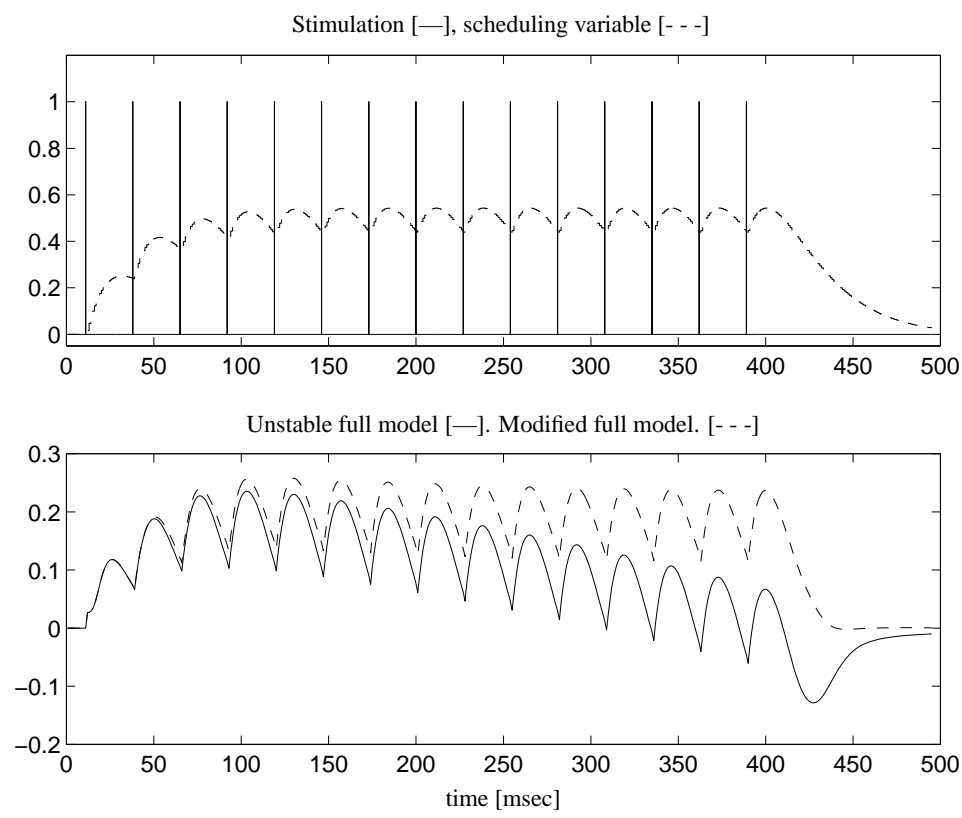

(a) Responses of the full models to constant frequency burst of $37 \mathrm{~Hz}$ (stimulation and scheduling variables shown in the upper plot).

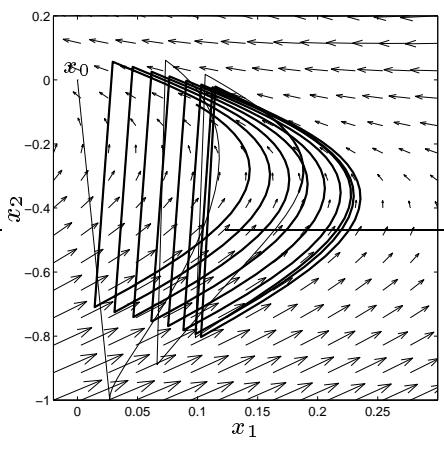

(b) Unstable local model.

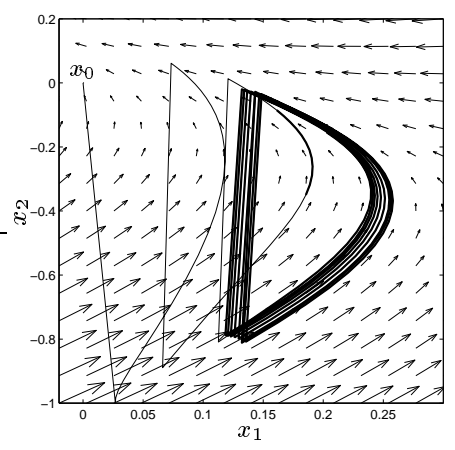

(c) Stable local model.

Figure 5.. Responses of the global models, and phase planes and state trajectories of the unstable and the stable local model for an input stimulation with a constant pulse frequency of $37 \mathrm{~Hz}$. The vertical jumps in the phase plots in 5(b) and 5(c) correspond to these pulse-like inputs. The trajectories are shown from 1 to $300 \mathrm{~ms}$, bold lines indicate that the fourth local model is active $\left(\rho_{4}>0.3\right)$. Note how the cycle in Figure 5(b) gradually drifts away, due to the effect of the slow unstable eigenvector. 
pole at -1 . After reidentifying the bias terms $d$ of the modified local model, it performs similarly to the previously identified model on the identification data, c.f. Figure 4(a), but does not become unbounded for the constant stimulation case. The full force-fields associated with the candidate local models are shown in Figure 6.

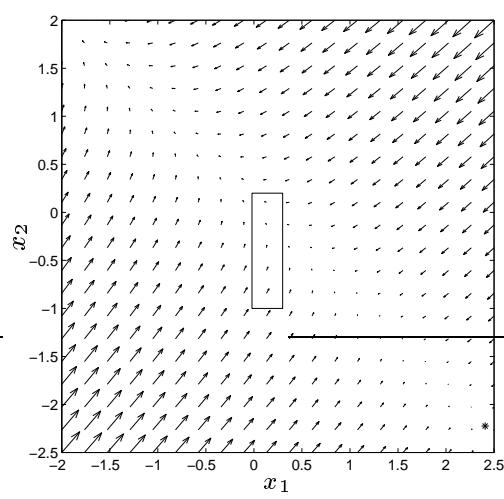

(a) Unstable local model.

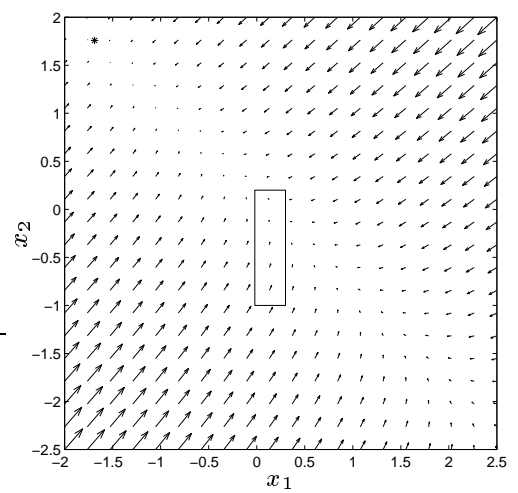

(b) Stable local model.

Figure 6.. Phase planes of the unstable and the stable local model. The $*$ denote the equilibrium points of the autonomous systems, $x_{4}^{e}=-A_{4}^{-1} d_{4}$. The rectangular area marks the region the local model operate in, c.f. Figure 5.

The above example clearly demonstrates the importance of understanding how a blended multiple model structure represents non-linear dynamics; namely that this understanding can be used to construct a global model with desired properties.

\section{Concluding remarks}

In this paper we have made the following important observations regarding the interpretation of local model dynamics in systems of blended local models:

(i) We illustrated that a genuine interpretation question arises when the model is identified from experimental data; namely does the identified model represent plant linearisations or global plant dynamics?

(ii) We have shown that the properties (the eigenspace) of the parameterised local models $\mathbf{A}(\mathbf{x}, \mathbf{u}, \mathbf{w})$ need not provide useful insight into the model dynamics, even in the neighbourhood of model equilibria. The extent to which the local models reflect actual plant dynamics depends on the offsets introduced by linearisation points, the form of the interpolation functions, and upon the location of these models with respect to the model equilibria. 
(iii) We have also demonstrated that a non-unique parameterisation of model dynamics exists off equilibria, or when offsets are identified from data. Hence, trying to interpret qualitatively the model dynamics based upon $\mathbf{A}_{\mathbf{i}}(\mathbf{x}, \mathbf{u}, \mathbf{w})$ off-equilibria is dangerous.

The muscle modelling and helicopter examples illustrated that these considerations are relevant for real applications, and that these effects can be used constructively to obtain 'well-behaved' local models. Furthermore, the muscle modelling example also illustrated that the ill-constrained nature of the identified local models can also be a problem, even if the model is used as a black-box structure, i.e. slight variations in identification data can lead to qualitatively different model behaviour. These problems will remain, due to the difficulties associated with experiment design. This can be somewhat constrained by separating the identification of local model offsets and $\mathbf{A}$ matrices, so that this case does not occur accidentally for on-equilibrium models. We can also use the non-unique parameterisation of the non-linear dynamics to construct global models from local models which are in some sense well behaved. Future regularisation-like approaches could provide a more general solution to apply in the identification stage.

Finally, we emphasise that the interpretation problems reported in this paper arise, not as a result of poor identification, but rather as a result of the nature of the multiple-model approach to building non-linear dynamical models. The authors believe that the full power of this approach will be realised only after the interpretation issue has been understood, and forms an integral part of the experiment design procedure.

\section{Acknowledgements}

Roderick Murray-Smith gratefully acknowledges the support of Marie Curie TMR grant FMBICT961369. Robert Shorten gratefully acknowledges the support of Forbairt and University College Dublin. The authors would like to especially thank Tor Arne Johansen, Daniel Sbarbaro-Hofer and Doug Leith for stimulating discussion relating to the material presented in this paper.

\section{REFERENCES}

Fillipov, A. F. (1960). Differential Equations With Discontinuous Right Hand Sides. Mathematicheskii Sbornik 51(1), 199-231.

Gollee, H., K.J. Hunt, N. Donaldson and J. Jarvis (1997). Modelling of electrically stimulated muscle. In: Multiple Model Approaches to Modelling and Control (R. Murray-Smith and T. A. Johansen, Eds.). Chap. 3, pp. 101-120. Taylor and Francis, London.

Houston, S. (1994). Validation of a non-linear individual blade rotorcraft flight dynamics model using a perturbation method. The Aeronautical Journal 98(977), 260-266.

Johansen, T. A. and R. Murray-Smith (1997). The operating regime approach to nonlinear modelling and control. In: Multiple Model Approaches to Modelling and Control 
(R. Murray-Smith and T. A. Johansen, Eds.). Chap. 1, pp. 3-72. Taylor and Francis, London.

Press, W. H., S. A. Teukolsky, W. T. Vetterling and B. P. Flannery (1992). Numerical Recipes $(C)$ : The Art of Scientific Computing. Chap. 15. 2nd ed.. Cambridge University Press.

Priestley, M. B. (1988). Non-linear and Non-stationary Time Series Analysis. Academic Press.

Shorten, R (1996). A Study of Hybrid Dynamical Systems with Application to Automotive Control. PhD thesis. Department of Electrical Engineering, University College Dublin, Republic of Ireland, June 1996.

Skoog, R. A. and G.Y. Clifford (1972). Instability of Slowly Varying Systems. IEEE Transactions on Automatic Control 17(1), 86-92.

Takagi, T. and M. Sugeno (1985). Fuzzy identification of systems and its applications for modeling and control. IEEE Trans. on Systems, Man and Cybernetics 15(1), 116132.

Tong, Howell (1990). Non-linear Time Series: A Dynamical System Approach. Oxford University Press. Oxford Statistical Science Series 6. 